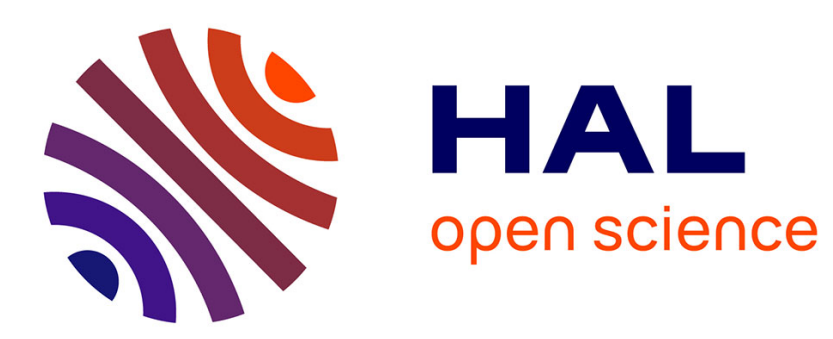

\title{
Rate of Convergence of a Numerical Procedure for Impulsive Control Problems
}

\author{
Mabel M. Tidball
}

\section{To cite this version:}

Mabel M. Tidball. Rate of Convergence of a Numerical Procedure for Impulsive Control Problems. RR-2926, INRIA. 1996. inria-00073772

\section{HAL Id: inria-00073772 \\ https://hal.inria.fr/inria-00073772}

Submitted on 24 May 2006

HAL is a multi-disciplinary open access archive for the deposit and dissemination of scientific research documents, whether they are published or not. The documents may come from teaching and research institutions in France or abroad, or from public or private research centers.
L'archive ouverte pluridisciplinaire HAL, est destinée au dépôt et à la diffusion de documents scientifiques de niveau recherche, publiés ou non, émanant des établissements d'enseignement et de recherche français ou étrangers, des laboratoires publics ou privés. 
INSTITUT NATIONAL DE RECHERCHE EN INFORMATIQUE ET EN AUTOMATIQUE

\section{Rate of convergence of a numerical procedure for impulsive control problems}

Mabel TIDBALL

$\mathbf{N}^{\circ} 2926$

July 1996

THÈME 4 



\title{
Rate of convergence of a numerical procedure for impulsive control problems
}

\author{
Mabel TIDBALL ${ }^{*}$ \\ Thème 4 - Simulation et optimisation \\ de systèmes complexes \\ Projet MIAOU \\ Rapport de recherche $\mathrm{n}^{\circ} 2926$ - July 1996 - 23 pages
}

\begin{abstract}
In this paper we consider a deterministic impulsive control problem. We discretize the Hamilton-Jacobi-Bellman equation satisfied by the optimal cost function and we obtain discrete solutions of the problem. We give an explicit rate of convergence of the approximate solutions to the solution of the original problem. We consider the optimal switching problem as a special case of impulsive control problem and we apply the same structure of discretization to obtain also a rate of convergence in this case. We present a numerical example.
\end{abstract}

Key-words: Impusive Control. Discretization. Hamilton-Jacobi-Bellman equations.

(Résumé : tsvp)

* Department of Mathematics, Universidad de Rosario, Pellegrini 250, 2000 Rosario, ARGENTINA.

Unité de recherche INRIA Sophia-Antipolis 2004 route des Lucioles, BP 93, 06902 SOPHIA-ANTIPOLIS Cedex (France)

Téléphone : (33) 93657777 - Télécopie : (33) 93657765 


\section{Vitesse de convergence pour un procedure numérique d'un problème de contrôle impultionnel}

Résumé : Dans ce papier nous considérons un problème de contrôle impulsionnel détérministe. Nous discrétisons l'équation de Hamilton-Jacobi-Bellman satisfaite par la fonction de coût optimale et nous trouvons les solutions discrètes de notre problème. Nous obtenons une expression explicite de la vitesse de convergence entre les solutions approchées et la solution du problème original. Nous considérons le problème de commutation optimale comme un cas particulier du problème de contrôle impulsionnel et nous appliquons la même structure de discrétisation pour avoir la vitesse de convergence dans ce cas. Nous présentons un exemple numérique.

Mots-clé : Contrôle impulsionnel. Discrétisation. Équations de Hamilton-Jacobi-Bellman 


\section{Introduction}

Optimization problems of dynamical systems lead to the treatment of non linear partial differential equations: the Hamilton-Jacobi-Bellman equations or Isaacs equations, associated to optimal control problems or differential game problems respectively. Except in special cases, the analytical solutions of these equations are unknown. Therefore, it is interesting to find characterisations of the existence, unicity, regularity of the solutions of these equations in order to develop numerical methods to obtain approximate solutions. With these solutions it is possible to obtain suboptimal feedback controls, equilibrium strategies, etc.

After the development of viscosity solutions (see [12], [25] and [16]), the approximation of Hamilton-Jacobi-Bellman equations was deeply studied.

The techniques to obtain numerical approximations are based on time-space discretizations. The time discretization procedure was introduced in [9], [11], for deterministic control problems (see also [2], [3], [5] for game problems with target) and the space discretization procedure uses finite elements techniques; see [22], [8] and [23] for control problems and [4] and [1] for game problems.

Another type of discretization, which involves only space discretization, was also studied in [19] and [28] for example.

In this paper we consider a deterministic implusive control problem (see [7]). We apply the time-space discretization procedure to obtain the discrete solutions.

It is organized as follows: In $\S 1$ we introduce the continuous problem and we give the properties of the optimal cost function as the unique viscosity solution of the associated Hamilton-Jacobi-Bellman equation (the proof is similar to the one presented in [10] for optimal switching problems).

In $\S 2$ we introduce a time discretization of the Hamilton-Jacobi-Bellman equation, we also give a convergence rate. The results obtained here are extensions of the results presented in [9] and [11] for impulsive control problems. The time discretization scheme involves a delay between impulsions. We can prove the existence and unicity of the discrete time solution because it is the fixed point of a contractive operator. We also define a non contractive scheme of time discretization. This scheme corresponds to a process where instantaneous impulsions are allowed. We prove that both schemes are equivalents.

In $\S 3$ we also discretize in the spatial variable in order to obtain an approximate problem whose solution can be found numerically. We give a rate of convergence of these solutions to the solution of the original problem.

In $\S 4$ we present a numerical example and finally, in $\S 5$, we consider the optimal switching problem (see [10]). We prove that this type of problem is a special case of impulsive control problems and we can apply the previously developed theory. In particular, in this case, due to the special structure of the optimal switching problem, we can obtain a better rate of convergence than the one obtained for general impulsive control problems.

$\mathrm{RR} \mathrm{n}^{\circ} 2926$ 


\section{Description of the continuous problem}

We consider a deterministic impulsive control problem, where the state of the system $y($.$) is$ given by the following ordinary differential equation:

$$
\frac{d y}{d s}(s)=g(y(s)) \quad s>0, \quad y(0)=x
$$

where $x \in \Omega, \Omega$ an open set of $\mathbb{R}^{n}$.

Equation (1) is valid $\forall s>0$ except at times $\theta_{\nu}$ where an impulsive control is applied. The impulsions are given by $z\left(\theta_{\nu}\right) \in Z, Z$ a compact set of $\mathbb{R}^{j}$, with $\theta_{\nu}<\theta_{\nu+1}$.

The impulsive controls, that we denote by $\mathbf{z}$, are determined by the sequence of values $\mathbf{z}()=.\left\{\left(\theta_{\nu}, z\left(\theta_{\nu}\right)\right), \nu=1,2 \ldots\right\}$. We call $\mathcal{Z}$ the set of impulsive controls.

The impulsions $z\left(\theta_{\nu}\right)$ produce a jump given by $\bar{g}\left(y\left(\theta_{\nu}^{-}\right), z\left(\theta_{\nu}\right)\right)$ that makes the system change instantaneously from position $y\left(\theta_{\nu}^{-}\right)$to $y\left(\theta_{\nu}^{+}\right)$; i.e.

$$
y\left(\theta_{\nu}^{+}\right)=y\left(\theta_{\nu}^{-}\right)+\bar{g}\left(y\left(\theta_{\nu}^{-}\right), z\left(\theta_{\nu}\right)\right)
$$

We assume that $y(s) \in \Omega \forall s>0$. The problem consists in finding the optimal cost function $u$, defined by:

$$
u(x)=\inf _{\mathbf{z}(.) \in \mathcal{Z}} J(x, \mathbf{z}(.)), \quad \forall x \in \mathbb{R}^{n}
$$

where

$$
J(x, \mathbf{z}(.))=\int_{0}^{\infty} f(y(s)) e^{-\lambda s} d s+\sum_{\nu=1}^{\infty} q\left(y\left(\theta_{\nu}^{-}, z\left(\theta_{\nu}\right)\right) e^{-\lambda \theta_{\nu}}\right.
$$

$f$ is the instantaneous cost, $\lambda>0$ is the discount rate and $q(x, z)$ is the cost of applying each impulsion.

\section{Properties of the optimal cost function $u$}

We assume that $\forall x, \tilde{x} \in \Omega, \forall z \in Z$ :

$$
\begin{aligned}
\bar{g}(x, .), q(x, .), \text { continuous } & \forall x \in \Omega \\
\|g(x)-g(\tilde{x})\| \leq L_{g}\|x-\tilde{x}\|, & \|g(x)\| \leq M_{g} \\
|f(x)-f(\tilde{x})| \leq L_{f}\|x-\tilde{x}\|, & |f(x)| \leq M_{f} \\
\|\bar{g}(x, z)-\bar{g}(\tilde{x}, z)\| \leq L_{\bar{g}}\|x-\tilde{x}\|, & \|\bar{g}(x, z)\| \leq M_{\bar{g}} \\
|q(x, z)-q(\tilde{x}, z)| \leq L_{q}\|x-\tilde{x}\|, & |q(x, z)| \leq M_{q} \\
q_{0}=\inf _{x, z} q(x, z)>0 &
\end{aligned}
$$

We call (H) the set of hypotheses (6) - (9). By virtue of (5), (H) and (10) we can prove (see [18]) that: 
Lemma 2.1 If a control $\mathbf{z}($.$) has more than \mu_{0}=\frac{2 e M_{f}}{q_{0} \lambda}$ impulsions in $[t, t+\delta)$, with $\delta=\frac{1}{\lambda}$ then there exists another control $\overline{\mathbf{z}}$ with cost strictly lower, i.e:

$$
J(x, \overline{\mathbf{z}})<J(x, \mathbf{z}) .
$$

Remark 2.1 By Lemma 2.1 we can deduce that for optimization purpose, we can consider only controls with at most $\mu_{0}$ impulsions in $[t, t+\delta)$. In consequence hereafter we shall assume that the controls verify this condition.

If we denote by $\mu(s)=\mu(s, \mathbf{z})$ the number of impulsions in $[0, s)$, with $\mu(\infty)$ the total number of impulsions of a generic control $\mathbf{z}($.$) , we have:$

$$
\mu(s) \leq \mu_{0}\left(1+\frac{s}{\delta}\right)
$$

If we call $\mu_{\delta}=\frac{\mu_{0}}{\delta}=\lambda \mu_{0}$, by virtue of (12) we have:

$$
\mu(s) \leq \mu_{0}+\mu_{\delta} s .
$$

As

$$
\nu-1=\mu\left(\theta_{\nu}\right) \leq \mu_{0}+\mu_{\delta} \theta_{\nu}
$$

and by equation (14), we have:

$$
\theta_{\nu} \geq \frac{\nu-1-\mu_{0}}{\mu_{\delta}}
$$

To obtain the regularity properties of the optimal cost function $u$, it is necessary to study the behavior of the trajectories as a function of the initial position. So we define:

$$
\lambda_{\bar{g}}=\sup \left\{\frac{\left\|x+\bar{g}(x, z)-x^{\prime}-\bar{g}\left(x^{\prime}, z\right)\right\|}{\left\|x-x^{\prime}\right\|}: z \in Z, x, x^{\prime} \in \Omega, x \neq x^{\prime}\right\} .
$$

By (8) we obtain that $\lambda_{\bar{g}} \leq 1+L_{\bar{g}}$. We can prove (see [18]), that:

Lemma 2.2 Under hypotheses (5) and $(H)$ we have:

$$
\left\|y(s)-y^{\prime}(s)\right\| \leq\left(\lambda_{\bar{g}}\right)^{\mu(s)} e^{L_{g} s}\left\|x-x^{\prime}\right\| .
$$

where $y, y^{\prime}$ are solutions of (1)-(2) for the same impulsive control, with initial condition $y(0)=x$ and $y^{\prime}(0)=x^{\prime}$.

Remark 2.2 By virtue of Lemma 2.2 we have that:

$$
\left\|y(s)-y^{\prime}(s)\right\| \leq \begin{cases}e^{L_{g} s}\left\|x-x^{\prime}\right\| & \text { if } \lambda_{\bar{g}} \leq 1, \\ \left(\lambda_{\bar{g}}\right)^{\mu_{0}} e^{\left(\mu_{\delta} \ln \lambda_{\bar{g}}+L_{g}\right) s}\left\|x-x^{\prime}\right\| & \text { if } \lambda_{\bar{g}}>1 .\end{cases}
$$

$\mathrm{RR} n{ }^{\circ} 2926$ 
Lemma 2.3 Under hypotheses (5), $(H)$ and (10), u satisfies:

$$
|u(x)| \leq \frac{M_{f}}{\lambda}, \quad\left|u(x)-u\left(x^{\prime}\right)\right| \leq C\left\|x-x^{\prime}\right\|^{\gamma} .
$$

For all $x, x^{\prime} \in \mathbb{R}^{n}$ and $\gamma=1$ if $\lambda>L, \gamma \in(0,1)$ if $\lambda=L, \gamma=\frac{\lambda}{L}$ if $\lambda<L$, with

$$
L=\left(\mu_{\delta} \ln \lambda_{\bar{g}}\right)^{+}+L_{g}
$$

For the proof see [29].

\section{The Hamilton-Jacobi-Bellman equation}

A function $u \in C\left(\mathbb{R}^{n}\right)$ is called a viscosity solution of the equation:

$$
\min \left\{\frac{\partial u}{\partial x} \cdot g+f-\lambda u, M u-u\right\}=0
$$

where

$$
M u(x)=\min _{z}\{u(x+\bar{g}(x, z))+q(x, z)\},
$$

if for all $\phi \in C^{1}\left(\mathbb{R}^{n}\right)$ satisfies:

i) $u-\phi$ has a local maximun in $x_{0}$, then $\min \left\{\frac{\partial \phi}{\partial x} \cdot g+f-\lambda u, M u-u\right\} \geq 0$ in $x_{0}$.

ii) $u-\phi$ has a local minimun in $x_{1}$, then $\min \left\{\frac{\partial \phi}{\partial x} \cdot g+f-\lambda u, M u-u\right\} \leq 0$ in $x_{1}$.

Employing the usual techniques and reazonig of dynamical programming (see [10]), we can prove:

Theorem 2.1 The optimal cost function $u$ is the unique solution, in the sense of viscosity of equation (19).

Remark 2.3 We can define the problem with finite horizon, that is:

$$
u_{T}(t, x)=\inf _{\bar{z}(.) \in \mathcal{Z}_{\mathcal{T}}} J_{T}(t, x, \bar{z}(.)), \quad \forall t \in[0, T], \forall x \in \mathbb{R}^{n} .
$$

where $\mathcal{Z}_{\mathcal{T}} \subset \mathcal{Z}$ such that $t \leq \theta_{1} \leq \ldots \leq \theta_{N} \leq T$.

$$
J_{T}(t, x, \bar{z}(.))=\int_{t}^{T} f(y(s)) e^{-\lambda s}+\sum_{\nu=1}^{N} q\left(y\left(\theta_{\nu}^{-}, z\left(\theta_{\nu}\right)\right) e^{-\lambda \theta_{\nu}}\right.
$$

INRIA 
$y($.$) is solution of:$

$$
\frac{d y}{d s}(s)=g(y(s)), s \in(t, T), \quad y(t)=x .
$$

If conditions (5), $(H)$ and (10) are satisfied and keeping (17) in mind we obtain that the optimal cost functions with finite horizon is bounded and Lipschitz continuous, i.e.

$$
\left|u_{T}(t, x)-u_{T}\left(t, x^{\prime}\right)\right| \leq \begin{cases}C\left|e^{(L-\lambda)(T-t)}-1\right|\left\|x-x^{\prime}\right\| & \text { if } L \neq \lambda \\ C(T-t) \mid\left\|x-x^{\prime}\right\| & \text { if } L=\lambda\end{cases}
$$

and

$$
\left|u(x)-u_{T}(0, x)\right| \leq C e^{-\lambda T} .
$$

The proofs are analogous to the one presented in [21].

\section{Time discretization of the Hamilton-Jacobi-Bellman equation}

\subsection{Time discretization scheme}

Let $h$ be suitably small. To find a discrete time approximation of (3) we consider the solution of

$$
u=T u
$$

where $T: X \longrightarrow X, X=C^{0, \gamma}$ with

$$
\|u\|_{\gamma}=\sup _{x}|u(x)|+\sup _{x_{1} \neq x_{2}} \frac{\left|u\left(x_{1}\right)-u\left(x_{2}\right)\right|}{\left|x_{1}-x_{2}\right|^{\gamma}}
$$

and $T$ defined in the following way:

$$
T=\min \left(P_{0}, P_{1}\right)
$$

where

$$
\begin{gathered}
P_{0} u(x)=(1-\lambda h) u(x+h g(x))+h f(x) \\
P_{1} u(x)=\min _{z}\{q(x, z)+h f(x+\bar{g}(x, z))+(1-\lambda h) u(x+\bar{g}(x, z)+h g(x+\bar{g}(x, z)))\} .
\end{gathered}
$$

$P_{0}$ and $P_{1}$ are time discretizations of (19). We can easily prove, following the same ideas of [11] that $P_{0}$ and $P_{1}$ are contractive operators, and so is $T$. Then we have the following Lemma:

Lemma 3.1 There exists a unique solution of (23) that we call $u^{h}$. 
Interpertation of $u^{h}$.

For all $x \in \mathbb{R}^{n}$ the following representation for $u^{h}$ is valid:

$$
u^{h}(x)=\min _{\mathbf{z}(.) \in \mathcal{Z}^{h}} J^{h}(x, \mathbf{z}(.))
$$

where:

$$
\begin{gathered}
\mathcal{Z}^{h}=\left\{\mathbf{z}(.) \in \mathcal{Z}: \theta_{i}=n_{i} h, n_{i} \in \mathbb{N}, n_{i}<n_{i+1}, i=1, \ldots\right\} \\
J^{h}(x, \mathbf{z}(.))=h \sum_{j=0}^{\infty} f\left(y_{h}^{+}(j)\right)(1-\lambda h)^{j}+\sum_{j=1}^{\infty} q\left(y_{h}^{-}\left(n_{j}\right), z_{j}\right)(1-\lambda h)^{n_{j}}
\end{gathered}
$$

Let $I(\mathbf{z}())=.\left\{j: \exists i / j=n_{i}\right\}$. We define la sequence $y_{h}(j)$ by the following recurrence formulae:

$$
\begin{cases}y_{h}^{-}(j+1)=y_{h}^{+}(j)+h g\left(y_{h}^{+}(j)\right) & \\ y_{h}^{+}\left(n_{j}\right)=y_{h}^{-}\left(n_{j}\right)+\bar{g}\left(y_{h}^{-}\left(n_{j}\right), z_{j}\right) & \text { if } j \in I(\mathbf{z}(.)) \\ y_{h}^{+}(j)=y_{h}^{-}(j) & \text { if } j \notin I(\mathbf{z}(.)) \\ y_{h}^{+}(0)=x & \end{cases}
$$

Remark 3.1 With the same arguments used in [18], we can prove that there exists an optimal discrete policy that realizes $u^{h}$, i.e., there exists $\tilde{\mathbf{z}}(.) \in \mathcal{Z}^{h}$ such that $u^{h}(x)=J^{h}(x, \tilde{\mathbf{z}}()$.$) .$ Moreover, we can prove (as in the continuous case), that the optimal policy has at most $\mu_{0}$ impulsions in each interval of lenght $\frac{1}{\lambda}$.

Remark 3.2 We can easily prove, by virtue of Remark 3.1 that $u^{h}$ is also a fixed point of operator $\bar{P}^{n}$, where:

$$
n=\left[\frac{1}{\lambda h}\right]
$$

$[x]$ represents the integer part of $x$.

$s=\left(s_{i}\right)_{1}^{n}$, with $s_{i} \in\{0,1\}, \quad|s|=\sum_{i=1}^{n} s_{i}, \quad \bar{S}=\left\{s ;|s| \leq \mu_{0}\right\}, \quad P_{s}=\prod_{1}^{n}\left(P_{0}\left(1-s_{i}\right)+P_{1} s_{i}\right)$

and

$$
\bar{P}^{n} w=\min _{s \in \bar{S}}\left(P_{s} w\right)
$$

We are going to use this remark latter.

Remark 3.3 We define function $u_{T}^{h}(n, x)$, the time discrete problem with finite horizon, for $h=\frac{T}{N}, N \in \mathcal{I N}, n=0, \ldots N$ (we suppose $h \in \mathcal{I N}$ ).

$$
u_{T}^{h}(n-1, x)=\min _{\mathbf{z}(.) \in \mathcal{Z}_{T}^{h}} J_{T}^{h}(n, x, \mathbf{z}(.))
$$

INRIA 
where $\mathcal{Z}_{T}^{h}=\left\{\mathbf{z}(.) \in \mathcal{Z}: \theta_{i}=n_{i} h, n_{i} \in \mathbb{N}, n_{i}<n_{i+1} \leq N, i=1, \ldots, N-1\right\}$

$$
J_{T}^{h}(n, x, \mathbf{z}(.))=h \sum_{j=0}^{N-1} f\left(y_{h}^{+}(j)\right)(1-\lambda h)^{j}+\sum_{j=1}^{N} q\left(y_{h}^{-}\left(n_{j}\right), z_{j}\right)(1-\lambda h)^{n_{j}}
$$

and the sequence $y_{h}(j)$ is given by (31) but now defined for all $1 \leq j \leq N-1$. We can obtain the following results:

$$
\left\|y_{h}(j)-\bar{y}_{h}(j)\right\| \leq C e^{L j h}\|x-\bar{x}\|,
$$

where $\bar{y}_{h}(j), j=1, \ldots, N-1$ is the solution of (31) with initial condition $\bar{x}$.

$$
u_{T}^{h}(n-1, x)=T\left(u_{T}^{h}(n, x)\right), \quad u_{T}^{h}(N, x)=0
$$

We will call $u_{T}^{h}(n, x)=u_{n}^{h}$, and $u_{T}^{h}(0, x)=u_{T}^{h}(x)$.

The function $u_{T}^{h}$ is Lipschitz continuous and its Lipschitz constant is bounded by:

$$
L_{u_{N}^{h}} \leq \begin{cases}C e^{(L-\lambda) T} & \text { if } L>\lambda \\ C & \text { if } L<\lambda \\ C T & \text { if } L=\lambda\end{cases}
$$

Moreover

$$
\left|u^{h}(x)-u_{T}^{h}(0, x)\right| \leq C e^{-\lambda T} .
$$

For the proof of these properties see [29].

The discrete solution $u^{h}$ has the following properties:

Lemma 3.2 Under hypotheses (5), (H) and (10), $u^{h}$ satisfies:

$$
\begin{gathered}
\left|u^{h}(x)\right| \leq \frac{M_{f}}{\lambda}, \quad\left|u^{h}(x)-u^{h}\left(x^{\prime}\right)\right| \leq C\left\|x-x^{\prime}\right\|^{\gamma} \\
\forall x, x^{\prime} \in \mathbb{R}^{n} \text { and } \gamma=1 \text { if } \lambda>L, \gamma \in(0,1) \text { if } \lambda=L, \gamma=\frac{\lambda}{L} \text { if } \lambda<L .
\end{gathered}
$$

The proof follows by the properties of function $u_{T}^{h}$ given in Remark 3.3.

Remark 3.4 From here, and in order to obtain simplicity of notation and clarity of arguments, we will use the letters $C, M, K$ to denote arbitrary constants, which values depends on the context where they appear, on the problem data (constants $\lambda, M_{g}, L_{g}, \ldots$ etc), but do not depend on the parameter of discretization $h$.

$\mathrm{RR} \mathrm{n}^{\circ} 2926$ 


\subsection{Rate of convergence of the $h$-approximate solution}

We want to find an estimate of the rate of convergence of $\left|u_{T}(x)-u_{T}^{h}(x)\right|$, so we consider the following auxiliary problem:

$$
u_{T}^{e, h}(x)=\min _{\mathbf{z}(.) \in \mathcal{Z}_{T}^{h}} J_{T}(x, \mathbf{z}(.))
$$

then

$$
\left|u_{T}(x)-u_{T}^{h}(x)\right| \leq\left|u_{T}(x)-u_{T}^{e, h}(x)\right|+\left|u_{T}^{e, h}(x)-u_{T}^{h}(x)\right|
$$

We will bound each term of (40). It is easy to prove that:

$$
\left|u_{T}^{e, h}(x)-u_{T}^{h}(x)\right| \leq C L_{u_{T}^{h}} h .
$$

This is just the estimate of the error associated to the Euler's integration method.

Now we must estimate $\left|u_{T}(x)-u_{T}^{e, h}(x)\right|$. Let $\mathbf{z} \in \mathcal{Z}_{T}$. It is necessary to obtain a policy $\overline{\mathbf{z}} \in \mathcal{Z}_{T}^{h}$ that approximates $\mathbf{z}$. Since the two parameters that determine $\overline{\mathbf{z}}$ are $\bar{\theta}_{\nu}$ and $\overline{\mathbf{z}}\left(\bar{\theta}_{\nu}\right)$, we consider the following definition for $\overline{\mathbf{z}}$ :

$$
\bar{\theta}_{\nu}=\max \left\{\bar{\theta}_{\nu-1}+h, h E\left(\frac{\theta_{\nu}}{h}\right)\right\}, \quad \bar{z}\left(\bar{\theta}_{\nu}\right)=z\left(\theta_{\nu}\right)
$$

where $\bar{\theta}_{0}=0, E(x)=[x]+1$ if $x \notin \mathcal{I N}$ and $E(x)=x$ if $x \in \mathcal{I N}$.

We call $\bar{y}($.$) the solution of (1) corresponding to control \overline{\mathbf{z}}($.$) .$

We estimate the difference $\|y(t)-\bar{y}(t)\|$. Let $n_{0}$ be such that till $n_{0} h$ there are no impulsions for $\overline{\mathbf{z}}($.$) and in \left(n_{0}+1\right) h, \ldots,\left(n_{0}+p\right) h$ there are impulsions, (we know that $p \leq \mu_{0}$ ). For the continuous time control $\mathbf{z}($.$) , there will exist \theta_{\nu_{j}+1}, \ldots, \theta_{\nu_{j}+p}$ such that:

$$
n_{0} h<\theta_{\nu_{j}+1} \leq\left(n_{0}+1\right) h, \quad \theta_{\nu_{j}+p} \leq\left(n_{0}+p\right) h .
$$

The worst case occurs when all the impulsions of the continuous control are in the first considered interval, i.e., when $\theta_{\nu_{j}+p} \leq\left(n_{0}+1\right) h$.

Lemma 3.3 Let $y(t)$ be the solution of (1) under the action of the control $\mathbf{z}($.$) and \bar{y}(t)$ the solution of (1) under the action of the approximate control $\overline{\mathbf{z}}($.$) defined in (42). Then, for$ all $t \notin \cap\left[\theta_{\nu}, \bar{\theta}_{\nu}\right]$ we have:

$$
\|y(t)-\bar{y}(t)\| \leq C h e^{L t}
$$

Proof: Let $t_{i}=i h$ and

$$
\tilde{y}_{r}=\bar{y}_{r-1}+\bar{g}\left(\tilde{y}_{r-1}, z\left(\theta_{\nu_{j}+r}\right)\right) \text {. }
$$

Then by (2)

$$
\left\|y\left(\theta_{\nu_{j}+r}^{+}\right)-\tilde{y}_{r}\right\| \leq \lambda_{\bar{g}}\left\|y\left(\theta_{\nu_{j}+r}^{-}\right)-\tilde{y}_{r-1}\right\| \leq \lambda_{\bar{g}}\left\|y\left(\theta_{\nu_{j}+r-1}^{+}\right)-\tilde{y}_{r-1}\right\|+C h .
$$

From this last inequality, it is easy to prove that:

$$
\left\|y\left(\theta_{\nu_{j}+p}^{+}\right)-\tilde{y}_{p}\right\| \leq\left(\lambda_{\bar{g}}\right)^{p}\left\|y\left(\theta_{\nu_{j}}^{+}\right)-\tilde{y}_{0}\right\|+C h,
$$


and the same argument is valid for $\bar{y}($.$) , i.e.:$

$$
\left\|\bar{y}\left(t_{n_{0}+p}^{+}\right)-\tilde{y}_{p}\right\| \leq\left(\lambda_{\bar{g}}\right)^{p}\left\|\bar{y}\left(t_{n_{0}}^{+}\right)-\tilde{y}_{0}\right\|+C h .
$$

Taking $\tilde{y}_{0}=y\left(\theta_{\nu_{j}}^{+}\right)$, we have:

$$
\left\|y\left(\theta_{\nu_{j}+p}^{+}\right)-\bar{y}\left(t_{n_{0}+p}^{+}\right)\right\| \leq\left(\lambda_{\bar{g}}\right)^{p}\left\|y\left(\theta_{\nu_{j}}^{+}\right)-\bar{y}\left(t_{n_{0}}^{+}\right)\right\|+C h
$$

From this inequality we obtain:

$$
\left\|y\left(t_{n_{0}+p}\right)-\bar{y}\left(t_{n_{0}+p}^{+}\right)\right\| \leq\left(\lambda_{\bar{g}}\right)^{p}\left\|y\left(t_{n_{0}}\right)-\bar{y}\left(t_{n_{0}}\right)\right\|+C h
$$

We define $E_{i}=\left\|y\left(t_{i}^{+}\right)-\bar{y}\left(t_{i}^{+}\right)\right\|$. Then From (44) we have:

$$
E_{n_{0}+p} \leq\left(\lambda_{\bar{g}}\right)^{p} E_{n_{0}}+C h
$$

When no impulsions occur we have:

$$
\begin{gathered}
E_{n_{0}+p+1} \leq E_{n_{0}+p}+\int_{t_{n_{0}+p}}^{t_{n_{0}+p+1}}(g(y(s))-g(\bar{y}(s))) d s \leq \\
\leq E_{n_{0}+p}+\int_{t_{n_{0}+p}}^{t_{n_{0}+p+1}}\left(g\left(y\left(t_{n_{0}+p}\right)\right)-g\left(\bar{y}\left(t_{n_{0}+p}\right)\right) d s+C h \leq\left(1+h L_{g}\right) E_{n_{0}+p}+C h .\right.
\end{gathered}
$$

From (45) we have:

$$
E_{n_{0}+p+1} \leq\left(1+h L_{g}\right)\left(\lambda_{\bar{g}}\right)^{p} E_{n_{0}}+C h
$$

Then, after the $n-\mu_{0}$ remaining intervals, we consider the worst case, i.e. $n_{0}=0$ and if we denote $n_{1}=n h$, we have:

$$
E_{n_{1}} \leq\left(1+h L_{g}\right)^{n-\mu_{0}}\left(\lambda_{\bar{g}}\right)^{p} E_{n_{0}}+C h
$$

By recurrence and considering that $E_{0}=0$, we have:

$$
E_{n_{1}} \leq\left\{\left(1+h L_{g}\right)^{n-\mu_{0}}\left(\lambda_{\bar{g}}\right)^{p}\right\}^{i \lambda}+C h
$$

Then by virtue of (46) we obtain $\forall t \notin \cap\left[\theta_{\nu}, \bar{\theta}_{\nu}\right]$ :

$$
\|y(t)-\bar{y}(t)\| \leq C h e^{L t}
$$

Remark 3.5 We can easily obtain that $u_{T}^{e, h}(x)-u_{T}(x) \leq C h L_{u_{T}^{h}}$. By $(H)$, (43) and the inequality $0 \leq \bar{\theta}_{\nu}-\theta_{\nu} \leq \mu_{0} h$, we have $u_{T}(x)-u_{T}^{e, h}(x) \leq 0$, then considering (40) and (41), we obtain:

$$
\left|u_{T}(x)-u_{T}^{h}(x)\right| \leq C L_{u_{T}^{h}} h
$$

RR n ${ }^{\circ} 2926$ 
Theorem 3.1 If (5), $(H)$ and (10) hold, then:

$$
\left|u(x)-u^{h}(x)\right| \leq C h^{\gamma}
$$

where $\gamma=1$ if $\lambda>L, \gamma \in(0,1)$ if $\lambda=L, \gamma=\frac{\lambda}{L}$ if $\lambda<L$.

Proof: Keeping in mind (47), and the fact that

$\left|u(x)-u^{h}(x)\right| \leq\left|u(x)-u_{T}(x)\right|+\left|u_{T}(x)-u_{T}^{h}(x)\right|+\left|u_{T}^{h}(x)-u^{h}(x)\right| \leq C e^{-\lambda T}+\left|u_{T}(x)-u_{T}^{h}(x)\right|$

we obtain an estimate that depends on $T$. Taking the minimun in $T$ we obtain the thesis.

Remark 3.6 We can work with a non contractive scheme of discretization. The time discretization of (19) can be understood as a problem where we allow simultaneous impulsions. We can consider the solution of:

$$
u=\bar{T} u
$$

where $\bar{T}: X \longrightarrow X$ is defined as $\bar{T}=\min \left(P_{0}, \bar{P}_{1}\right), P_{0}$ is defined in (26) and

$$
\bar{P}_{1} u(X)=\min _{z}(q(x, z)+u(x+\bar{g}(x, z)))
$$

By using mainly the hypotheses (10) and the theory of B-L (Bensoussan-Lions) algorithm, introduced in [24] and the techniques decribed in [20], we obtain that there exists a unique solution of (48) that we call $\bar{u}^{h}$. Moreover we have that problems (23) and (48) are equivalent in the following sense:

$$
0 \leq u^{h}-\bar{u}^{h} \leq C h^{\gamma}
$$

For the proof see [29].

\section{The fully discrete solution of HJB equation}

\subsection{Description of the fully discrete problem}

The above introduced time discretization remains a theoretical one. To obtain computational results it is also necessary to perform a space discretization. We will use the same discretization as the one introduced in [13], [14], [19], [22] and [27]. Let $\Omega$ be an open set of $\mathbb{R}^{n}$ and $S_{j}^{k}$ a family of regular triangulations of $\Omega$, we define $\Omega_{k}=\cup_{j} S_{j}^{k}, k=\max _{j}\left(\operatorname{diam} S_{j}^{k}\right)$. Let $W_{k}: \Omega_{k} \longrightarrow \mathbb{R}$ be the set of finite linear elements. Then, the fully discrete problem is:

\section{Problem $P_{k}:$ Find the fixed point of operator $T$ in $W_{k}$}

We understand operator $T$ (see $P_{0}$ and $P_{1}$ definitions) as an operator $T: W^{k} \rightarrow W^{k}$, thats means we understand, for example, $u(x+h g(x))=\sum_{j} \lambda_{i j} u\left(x_{j}\right)$ where $x_{j}, j=1, \ldots, \bar{N}$ is the set of nodes of the triangulation and $\lambda_{i j}$ the baricentric coordenates such that $x+h g(x)$ belongs to the simplex $S_{j}$. 
Theorem 4.1 There exists a unique solution of problem $P_{k}$ that we call $u_{k}^{h}$.

Remark 4.1 To obtain $u_{k}^{h}$ it is only necessary to compute $u_{k}^{h}\left(x_{i}\right), i=1, \ldots, \bar{N}$, where $\left\{x_{i}: i=1, \ldots, \bar{N}\right\}$ is the set of nodes of the triangulation.

\section{Definitions and auxiliary results}

Let $\beta_{1}(.) \in C^{\infty}\left(\mathbb{R}^{n}\right), \beta_{1}(x) \geq 0 \forall x$, support of $\beta_{1} \subset B_{1}=\left\{x \in \mathbb{R}^{n}:\|x\| \leq 1\right\}$, $\int_{\mathbb{R}^{n}} \beta_{1}(x) d x=1, \beta_{\rho}(x)=\frac{1}{\rho^{n}} \beta_{1}\left(\frac{x}{\rho}\right), \rho \in \mathbb{R}^{+}$.

We define $u_{n, \rho}^{h}$ and $\tilde{u}_{n, \rho}^{h}$, the regularized function of $u_{n}^{h}$ and its linear interpolation respectively, in the following way:

$$
u_{n, \rho}^{h}(x)=\left(u_{n}^{h} * \beta_{\rho}\right)(x)=\int_{B(\rho)} u_{n}^{h}(x-y) \beta_{\rho}(y) d y
$$

We define $\tilde{u}_{n, \rho}^{h}$ the element of $W_{k}$ such that $\tilde{u}_{n, \rho}^{h}\left(x_{i}\right)=u_{n, \rho}^{h}\left(x_{i}\right), \forall i=1, \ldots, \tilde{N}$.

We easily obtain that these functions are Lipschitz continuous, with Lipschitz constant $L_{u_{n}^{h}}$.

We define recursively $u_{k, n}^{h}$ the fully discrete optimal cost function for the problem with finite horizon, $1 \leq n \leq N=\frac{T}{h}$, in the following way: $u_{k, n}^{h} \in W_{k}$ and

$$
u_{k, n-1}^{h}=T u_{k, n}^{h}, \quad u_{k, N}^{h}=0
$$

and we will denote $u_{k, N}^{h}=u_{k, 0}^{h}$.

We are going to obtain an estimate of the difference between the time discrete solution and the fully discrete solution. The following two Lemmas are devoted to prove that $u_{n}^{h}$ defined in (36) is "almost" a subsolution for the fully discrete problem.

Lemma 4.1 if (5), $(H)$ and $(10)$ are valid, then:

$$
\hat{u}_{n, \rho}^{h}\left(x_{i}\right) \leq u_{n, k}^{h}\left(x_{i}\right) \quad \forall x_{i}
$$

where $\hat{u}_{n, \rho}^{h}=-p\left(\frac{2 M_{f}}{\lambda+1}+(1-p) \tilde{u}_{n, \rho}^{h}\right.$ and

$$
p=\rho \max \left\{\frac{L_{f}\left(1+L_{\bar{g}}\right)+L_{g} L_{u_{n+1}^{h}}}{\lambda\left(1+\frac{M_{f}}{\lambda}\right)}+C L_{u_{n+1}^{h}} \frac{k^{2}}{h \rho^{2}}, \frac{L_{q}+L_{\bar{g}} L_{u_{n+1}^{h}}}{q_{0}}\right\}
$$

Proof: From (36) we know that:

$u_{n}^{h}(x) \leq\left\{\begin{array}{l}(1-\lambda h) u_{n+1}^{h}(x+h g(x))+h f(x) \\ \min _{z}\left\{q(x, z)+h f(x+\bar{g}(x, z))+(1-\lambda h) u_{n+1}^{h}(x+\bar{g}(x, z)+h g(x+\bar{g}(x, z)))\right\}\end{array}\right.$ 
By the definition of convolution and by the last inequality, we obtain:

$$
u_{n, \rho}^{h}(x) \leq\left\{\begin{aligned}
&(1-\lambda h) u_{n+1, \rho}^{h}(x+h g(x))+h f(x)+h \rho\left(L_{f}+L_{g} L_{u_{n+1}^{h}}\right) \\
& \min _{z}\{q(x, z)+h f(x+\bar{g}(x, z)) \\
&\left.+(1-\lambda h) u_{n+1, \rho}^{h}(x+\bar{g}(x, z)+h g(x+\bar{g}(x, z)))\right\} \\
&+h \rho\left(L_{f}\left(1+L_{\bar{g}}\right)+L_{g} \lambda_{\bar{g}} L_{u_{n+1}^{h}}\right)+\rho\left(L_{q}+L_{\bar{g}} L_{u_{n+1}^{h}}^{h}\right)
\end{aligned}\right.
$$

The function $u_{n, \rho}^{h}$ has second derivatives bounded by:

$$
\left\|D^{2} u_{n . \rho}^{h}\right\| \leq C \frac{L_{u_{n}^{h}}}{\rho}
$$

where $C$ is a constant, (because $u_{n, \rho}^{h}$ is the regularization of a Lipschitz function). Then, (see [19]), we can bound the difference between $u_{n, \rho}^{h}$ and its linear interpolation $\tilde{u}_{n, \rho}^{h}$ in the following way:

$$
\left\|u_{n, \rho}^{h}-\tilde{u}_{n . \rho}^{h}\right\| \leq C L_{u_{n}^{h}} \frac{k^{2}}{\rho}
$$

then by (51) we have:

$$
\tilde{u}_{n, \rho}^{h}(x) \leq\left\{\begin{aligned}
(1-\lambda h) \tilde{u}_{n+1, \rho}^{h}(x+h g(x))+h f(x)+h \rho\left(L_{f}+L_{g} L_{u_{n+1}^{h}}\right)+C L_{u_{n+1}^{h}} \frac{k^{2}}{\rho} \\
\min _{z}\{q(x, z)+h f(x+\bar{g}(x, z)) \\
\left.+(1-\lambda h) \tilde{u}_{n+1, \rho}^{h}(x+\bar{g}(x, z)+h g(x+\bar{g}(x, z)))\right\} \\
+h \rho\left(L_{f}\left(1+L_{\bar{g}}\right)+L_{g} \lambda_{\bar{g}} L_{u_{n+1}^{h}}\right)+\rho\left(L_{q}+L_{\bar{g}} L_{u_{n+1}^{h}}\right)+C L_{u_{n+1}^{h}} \frac{k^{2}}{\rho}
\end{aligned}\right.
$$

Defining

$$
\hat{u}_{n, \rho}^{h}=-p\left(\frac{2 M_{f}}{\lambda}+1\right)+(1-p) \tilde{u}_{n, \rho}^{h}
$$

from (53), we have:

$$
\tilde{u}_{n, \rho}^{h}(x) \leq\left\{\begin{array}{c}
(1-\lambda h) \tilde{u}_{n+1, \rho}^{h}(x+h g(x))+h f(x)+h \rho\left(L_{f}+L_{g} L_{u_{n+1}^{h}}\right) \\
+C L_{u_{n+1}^{h}} \frac{k^{2}}{\rho}-p \lambda h\left(1+\frac{M_{f}}{\lambda}\right. \\
\min _{z}\left\{q(x, z)+h f(x+\bar{g}(x, z))+(1-\lambda h) \tilde{u}_{n+1, \rho}^{h}(x+\bar{g}(x, z)+h g(x+\bar{g}(x, z)))\right\} \\
+h \rho\left(L_{f}\left(1+L_{\bar{g}}\right)+L_{g} \lambda_{\bar{g}} L_{u_{n+1}^{h}}\right)+\rho\left(L_{q}+L_{\bar{g}} L_{u_{n+1}^{h}}\right)+C L_{u_{n+1}^{h}} \frac{k^{2}}{\rho} \\
-p \lambda h\left(1+\frac{M_{f}}{\lambda}-p q_{0}\right.
\end{array}\right.
$$

Then, if we define:

$$
p=\rho \max \left\{\frac{L_{f}\left(1+L_{\bar{g}}\right)+L_{g} L_{u_{n+1}^{h}}}{\lambda\left(1+\frac{M_{f}}{\lambda}\right)}+C L_{u_{n+1}^{h}} \frac{k^{2}}{h \rho^{2}}, \frac{L_{q}+L_{\bar{g}} L_{u_{n+1}^{h}}}{q_{0}}\right\}
$$

we obtain:

$\tilde{u}_{n}^{h}(x) \leq\left\{\begin{array}{l}(1-\lambda h) \tilde{u}_{n+1}^{h}(x+h g(x))+h f(x) \\ \min _{z}\left\{q(x, z)+h f(x+\bar{g}(x, z))+(1-\lambda h) \tilde{u}_{n+1}^{h}(x+\bar{g}(x, z)+h g(x+\bar{g}(x, z)))\right\}\end{array}\right.$ 
in consequence $\tilde{u}_{n, \rho}^{h}$ is a subsolution of (49) and $\tilde{u}_{n, \rho}^{h} \leq u_{n, k}^{h}$.

Lemma 4.2 If (5), $(H)$ and (10) are valid, then for all $n=1, \ldots, \tilde{N}$, we have:

$$
u_{n}^{h}\left(x_{i}\right) \leq u_{n, k}^{h}\left(x_{i}\right)+C L_{u^{h} n+1}^{h} \frac{k}{\sqrt{h}}
$$

Proof: Taking in definition (50) $\rho=\frac{k}{\sqrt{h}}$ we obtain $p=C L_{u_{n+1}^{h}} \frac{k}{\sqrt{h}}$, so:

$$
(1-p) \tilde{u}_{n, \rho}^{h}\left(x_{i}\right) \leq u_{n, k}^{h}\left(x_{i}\right)+p\left(2 M_{f}+1\right)
$$

that implies:

$$
\tilde{u}_{n, \rho}^{h}\left(x_{i}\right) \leq u_{n, k}^{h}\left(x_{i}\right)+C L_{u_{n+1}^{h}} \frac{k}{\sqrt{h}}
$$

Then, by definition of $\tilde{u}_{n, \rho}^{h}$, we obtain:

$$
u_{n, \rho}^{h}\left(x_{i}\right) \leq u_{n, k}^{h}\left(x_{i}\right)+C L_{u_{n+1}^{h}} \frac{k}{\sqrt{h}}
$$

finally, keeping in mind that:

$$
\left|u_{n}^{h}(x)-u_{n, \rho}^{h}(x)\right| \leq L_{u_{h}^{n} \rho}
$$

we obtain:

$$
u_{n}^{h}\left(x_{i}\right) \leq u_{n, k}^{h}\left(x_{i}\right)+C L_{u_{n+1}^{h}} \frac{k}{\sqrt{h}}
$$

The following two Lemmas are devoted to prove that $u_{n}^{h}$ is "almost" a supersolution for the fully discrete problem.

Lemma 4.3 Let $n h=\frac{1}{\lambda}, T=N h, E_{N}=0$ and assume that in each interval of length $h$, relation (54) is verified at most $\mu_{0}$ times and relation (55) holds in the remaining points.

$$
\begin{aligned}
E_{i} & \leq(1-\lambda h) E_{i+1}+C L_{u_{i+1}^{h}} \frac{k}{\sqrt{h}} \\
E_{i} & \leq(1-\lambda h) E_{i+1}+C L_{u_{i+1}^{h}} k \sqrt{h}
\end{aligned}
$$

then:

$$
E_{0} \leq M \frac{k}{\sqrt{h}}
$$

RR n ${ }^{\circ} 2926$ 
For the proof see [29].

Lemma 4.4 If (5), $(H)$ and (10) are valid, then, $\forall x_{i}$ :

$$
u_{n, k}^{h}\left(x_{i}\right) \leq u_{n}^{h}\left(x_{i}\right)+C e^{(L-\lambda) T} \frac{k}{\sqrt{h}}
$$

Proof: As $u_{n}^{h}$ corresponds to the optimization porblem (36), there exists $\bar{s}$, with $|\bar{s}| \leq \mu_{0} \lambda T$, such that $u_{0}^{h}=P_{\bar{s}} u_{N}^{h}$. Calling $\nu_{1}, \ldots, \nu_{\bar{\mu}(h, T)}$ the indices $j$ such that $s_{j}=1$ we have that for such indices the following relations hold:

$$
\begin{array}{r}
\tilde{u}_{n, \rho}^{h} \geq\left\{q(x, z)+h f(x+\bar{g}(x, z))+(1-\lambda h) \tilde{u}_{n+1, \rho}^{h}(x+\bar{g}(x, z)+h g(x+\bar{g}(x, z)))\right\} \\
-h \rho\left(L_{f}\left(1+L_{\bar{g}}\right)+L_{g} \lambda_{\bar{g}} L_{u_{n+1}^{h}}\right)-\rho\left(L_{q}+L_{\bar{g}} L_{u_{n+1}^{h}}\right)-C L_{u_{n+1}^{h}} \frac{k^{2}}{\rho} \\
u_{n, k}^{h} \leq q(x, z)+h f(x+\bar{g}(x, z))+(1-\lambda h) \tilde{u}_{n+1, \rho}^{h}(x+\bar{g}(x, z)+h g(x+\bar{g}(x, z)))
\end{array}
$$

and for $j$ such that $s_{j}=0$ the following relations hold:

$$
\begin{gathered}
\tilde{u}_{n, \rho}^{h} \geq h f(x)+(1-\lambda h) \tilde{u}_{n+1, \rho}^{h}(x+h g(x))-h \rho\left(L_{f}\left(1+L_{\bar{g}}\right)+L_{g} \lambda_{\bar{g}} L_{u_{n+1}^{h}}\right)-C L_{u_{n+1}^{h}} \frac{k^{2}}{\rho} \\
u_{n, k}^{h} \leq(1-\lambda h) \tilde{u}_{n+1, \rho}^{h}(x+h g(x))+h f(x)
\end{gathered}
$$

If we define $E_{n}=\max _{i}\left\{u_{n, k}^{h}\left(x_{i}\right)-\tilde{u}_{n, \rho}^{h}\left(x_{i}\right)\right\}$, from (57) and (58) for $j$ such that $s_{j}=1$ we obtain, minimizing in $\rho$, that:

$$
E_{n} \leq(1-\lambda h) E_{n+1}+C L_{u_{n+1}^{h}} \frac{k}{\sqrt{h}}
$$

while for $j$ such that $s_{j}=0$, by relations (59) and (60) we obtain

$$
E_{n} \leq(1-\lambda h) E_{n+1}+C L_{u_{n+1}^{h}} k \sqrt{h}
$$

then,by Lemma 4.3 we obtain

$$
E_{0} \leq M \frac{k}{\sqrt{h}}
$$

Then:

$$
\begin{array}{r}
u_{n, k}^{h} \leq \tilde{u}_{n, \rho}^{h}+C e^{(L-\lambda) T} \frac{k}{\sqrt{h}} \leq u_{n}^{h}+\left|\tilde{u}_{n, \rho}^{h}-u_{n, \rho}^{h}\right|+\left|u_{n, \rho}^{h}-u_{n}^{h}\right|+C e^{(L-\lambda) T} \frac{k}{\sqrt{h}} \leq \\
\leq u_{n}^{h}+C e^{(L-\lambda) T} \frac{k^{2}}{\rho}+C e^{(L-\lambda) T} \rho+C e^{(L-\lambda) T} \frac{k}{\sqrt{h}} \leq u_{n}^{h}+C e^{(L-\lambda) T} \frac{k}{\sqrt{h}}
\end{array}
$$


Remark 4.2 We have just obtain the rate of convergence for the problem with finite horizon. Indeed if (5), $(H)$ and (10) hold, then:

$$
\left|u_{T}^{h}(x)-u_{k, T}^{h}(x)\right| \leq C e^{(L-\lambda) T} \frac{k}{\sqrt{h}} .
$$

The proof is obvious by virtue of Lemma 4.2 and Lemma 4.4 .

\subsection{Rate of convergence of the fully discrete solution}

Theorem 4.2 If (5), $(H)$ and (10) hold, then:

$$
\left|u(x)-u_{k}^{h}(x)\right| \leq C\left(h+\frac{k}{\sqrt{h}}\right)^{\gamma}
$$

where $\gamma=1$ if $\lambda>L, \gamma \in(0,1)$ if $\lambda=L, \gamma=\frac{\lambda}{L}$ if $\lambda<L$.

Proof: The proof is evident by virtue of the following inequality and Remark 4.2.

$$
\begin{array}{r}
\left|u(x)-u_{k}^{h}(x)\right| \leq\left|u(x)-u_{T}(x)\right|+\left|u_{T}(x)-u_{T}^{h}(x)\right| \\
+\left|u_{T}^{h}(x)-u_{k, T}^{h}(x)\right|+\left|u_{k, T}^{h}(x)-u_{k}^{h}(x)\right| \leq \\
\leq C e^{-\lambda T}+\left|u_{T}(x)-u_{T}^{h}(x)\right|+\left|u_{T}^{h}(x)-u_{k, T}^{h}(x)\right|
\end{array}
$$

Remark 4.3 In the usual case, i.e. when $h$ is the same order than $k$ (which means when there exists $m_{1}$ and $m_{2}$ such that $m_{1} h \leq k \leq m_{2} h$ ), formula (61) becomes:

$$
\left|u(x)-u_{k}^{h}(x)\right| \leq M k^{\frac{\gamma}{2} \wedge \frac{1}{2}}
$$

Remark 4.4 Optimising (61) in $h$, we obtain the optimal value for $h=k^{\frac{2}{3}}$ and then:

$$
\left|u(x)-u_{k}^{h}(x)\right| \leq M k^{\frac{2 \gamma}{3} \wedge \frac{2}{3}}
$$

\section{$5 \quad$ Numerical example}

We consider a problem where $\Omega=(0,2), f(x)=-x, q(x, z)=q_{0}$, and the dynamics of the system given by:

$$
\frac{d y}{d s}=-b y(s), \quad y(0)=x, \quad b>0
$$

while no impulsive control is applied. When an impulsion is applied the jump is given by:

$$
\bar{g}(y)= \begin{cases}1 & \text { if } 0<y<1 \\ 2-y & \text { if } 1 \leq y<2\end{cases}
$$

$\mathrm{RR} \mathrm{n}^{\circ} 2926$ 
In this case we can find the analytical solution of the problem. If we call $\xi$ the point where the system jumps the solution is given by:

$$
u(x)= \begin{cases}u(2)\left(\frac{2}{x}\right)^{\frac{\lambda}{b}}-\frac{x-2\left(\frac{2}{x}\right)^{\frac{\lambda}{b}}}{\lambda+b} & \text { if } \xi<x \leq 2 \\ u(2)+q_{0} & \text { if } 1<x \leq \xi \\ u(x+1)+q_{0} & \text { if } 0<x \leq 1\end{cases}
$$

Remark $5.1 \xi$ is given by a relation between the data problem. In effect of (62), we obtain:

$$
u(2)=u 2(\xi)=\frac{-\frac{\xi-(2 / \xi)^{\lambda / b}}{\lambda+b}-q_{0}}{1-(2 / \xi)^{\lambda / b}}
$$

( $\xi$ is given by the greasted point where the last equality is valid).

Remark 5.2 The structure of an optimal feedback policy is the following: $(\xi, 2)$ is the set of continuation; $(0, \xi)$ is the set of application of impulsive control.

In this example, with $\lambda=0.5, q_{0}=0.5, b=0.125$, we obtain $\xi=1.5$ and $u(2)=-3.125$. Table 2 gives us the maximun error between the real and the approximate solution for different values of $\bar{N}$ using space discretization $h=k$. Table 1 shows the same measure using $h=k^{\frac{2}{3}}$.

\begin{tabular}{clcl}
\multicolumn{2}{c}{ Table 1 } & \multicolumn{2}{c}{ Table 2 } \\
$\bar{N}$ & error & $\bar{N}$ & error \\
80 & 0.24473 & 80 & 0.076639 \\
160 & 0.01215 & 160 & 0.04788 \\
320 & 0.0060813 & 320 & 0.030016 \\
640 & 0.0029928 & 640 & 0.018822 \\
1280 & 0.0014999 & 1280 & 0.011816
\end{tabular}

\section{$6 \quad$ Optimal switching problems}

\subsection{Description of the problem}

We consider in this section a deterministic optimal switching problem, (see [10]), of a system described by an ordinary differential equation, which dynamics can be modified, at the price of a positive switching cost, into anyone of a different setting.

The problem consists in finding the optimal way to modify the dynamics with the purpose of minimizing an associated cost. 
More precisely, let us define an admissible control $\alpha()=.\left\{\left(\theta_{\nu}, d_{\nu}\right): \nu=1,2, \ldots\right\}$ to be a sequence of switching times $\theta_{\nu}$ and control setting or switching decisions $d_{\nu-1} \longrightarrow d_{\nu}$, where

$$
\theta_{\nu} \in[0,+\infty), \theta_{\nu}<\theta_{\nu+1} \quad d_{\nu} \in D=\{1, \ldots, m\}
$$

then, the control $d($.$) remains constant in each interval \left[\theta_{\nu}, \theta_{\nu+1}\right)$ being:

$$
d(t)= \begin{cases}d \in D & \text { if } 0 \leq t<\theta_{1} \\ d_{\nu} \in D & \text { if } \theta_{\nu} \leq t<\theta_{\nu+1}, \quad \nu \geq 1\end{cases}
$$

For each $d \in D$ we also define $A^{d}$, the set of all admissible controls with initial setting $d$. For a given $x \in \mathbb{R}^{n}, d \in D, \alpha \in A^{d}$, the response of the system to the control $\alpha($.$) is given$ by the following ordinary differential equation:

$$
\frac{d y}{d s}=g\left(y(s), d_{\nu}\right), \theta_{\nu} \leq t<\theta_{\nu+1} \quad y(0)=x
$$

Our goal is to desing for each $x \in \mathbb{R}^{n}, d \in D$, an optimal control $\bar{\alpha}$, such that:

$$
u^{d}(x)=\inf _{\alpha \in A^{d}} J^{d}(x, \alpha)=J^{d}(x, \bar{\alpha})
$$

where

$$
J^{d}(x, \alpha)=\sum_{\nu=1}^{\infty}\left\{\int_{\theta_{\nu-1}}^{\theta_{\nu}} f\left(y(s), d_{\nu-1}\right) e^{-\lambda s} d s+q\left(d_{\nu-1}, d_{\nu}\right) e^{-\lambda \theta_{\nu}}\right\}
$$

$f: \mathbb{R}^{n} \times D \longrightarrow \mathbb{R}$ is the instantaneous cost and $q(d, \tilde{d})$ is the transition cost to replace $d$ by $\tilde{d}$. Besides the usual assumptions of regularity and boundedness for $f, g$ and $q$, we assume that:

$$
q(d, \tilde{d}) \geq q_{0}>0, \quad q(d, \tilde{d}) \leq q(d, \hat{d})+q(\hat{d}, \tilde{d})
$$

We understand this last inequality as follows: it is always cheaper to switch directly from setting $d$ to setting $\tilde{d}$ than to switch though an intermediate setting $\hat{d}$.

Remark 6.1 We can prove following a reasoning analogous at [10] that there exists an optimal switching control with a simple structure in terms of feedback policies. The key to the proof consists in showing that there exists $\sigma>0$ such that $\theta_{i} \geq \theta_{i-1}+\sigma$.

Remark 6.2 We can prove, (see [10]), that the set of inequalities (63) can be replaced by: $\exists q_{0}>0$ such that for all closed sequences of indices $d_{0}, \ldots, d_{n}$, with $d_{0}=d_{n}$, we have:

$$
q\left(d_{0}, d_{1}\right)+q\left(d_{2}, d_{3}\right)+\ldots+q\left(d_{n-1}, d_{n}\right) \geq q_{0}
$$

obtaining in this case the existence of the optimal cost function.

$\operatorname{RR} n^{\circ} 2926$ 


\subsection{Equivalent impulsive problem}

To obtain a fully discrete solution of the optimal switching problem and to estimate the rate of convergence towards the real solution, we are going to prove that this type of problem is equivalent to a special impulsive control problem.

We consider the following generalized version of the impulsive control problem presented in $\S 1$, extended for $\Omega=D \times \mathbb{R}^{n}$, where the evolution of the system takes the form:

$$
(d, y)(s)=\left(d_{\nu}, y\left(\theta_{\nu}^{+}\right)+\int_{\theta_{\nu}}^{s} g\left(y(t), d_{\nu}\right) d t\right)
$$

valid for $\theta_{\nu} \leq s<\theta_{\nu+1}$.

The jumps of the generalized state at instant $\theta_{\nu}$ concern only the first component of the state, i.e.

$$
\left(\tilde{d}, y\left(\theta_{\nu}\right)\right)=\left(d, y\left(\theta_{\nu}\right)\right)+(\tilde{d}-d, 0)
$$

Remark 6.3 It is easy to see that $\lambda_{\bar{g}}=1$. that is, in this case by (18) $L=L_{g}$.

It is evident that optimal switching problems can be considered as particular cases of impulsive control problems, where the dynamics associated to the impulsive part is not expansive. So we can anounce the following results:

$$
\min \left\{\frac{\partial u^{d}}{\partial x} \cdot g+f-\lambda u^{d}, M u^{d}-u^{d}\right\}=0
$$

where

$$
M u^{d}(x)=\min _{d \neq \tilde{d}}\left\{u^{\tilde{d}}(x)+q(d, \tilde{d})\right\}
$$

and the following rate of convergence of the discrete solution is valid:

$$
\left|u^{d}(x)-u_{k}^{d, h}(x)\right| \leq C\left(h+\frac{k}{\sqrt{h}}\right)^{\gamma}
$$

where $\gamma=1$ if $\lambda>L_{g}, \gamma \in(0,1)$ if $\lambda=L_{g}, \gamma=\frac{\lambda}{L_{g}}$ if $\lambda<L_{g}$. Note that this last inequality give a smaller estimate than (61) because $L=L_{g}$.

\section{Conclusions}

We have studied the fully discrete solution of an impulsive control problem. We have obtained the rate of convergence of the discrete solutions to the real solution, with a delay scheme and with a scheme that considers instantaneous impulsions. We have obtained an estimate of type $\left(h+\frac{k}{\sqrt{h}}\right)^{\gamma}$. When $h$ is of order $k$ and $\gamma=1$, that is when $u$ is Lipschitz continuous, we have obtained an estimate of type $k^{\frac{1}{2}}$, which improves the estimate obtained in [15] and

INRIA 
[19], where they obtain an estimate of type $k^{\frac{1}{2}}|\ln (k)|$.

When we have done the time optimization with respect to $h$, considering fixed $k$, we have obtained a bound that depends only on the parameter $k$ of order $k^{\frac{2 \gamma}{3}} \wedge \frac{2}{3}$.

We have also proved that the optimal switching problem is a special case of an impulsive control problem.

We have developed a simple numerical example, where the exact solution is known, in order to show the error between the real and the approximate solution for different relations between the time and the space discretization.

\section{References}

[1] Alziary de Roquefort B., "Jeux différentiels et approximation numériques de fonctions valeur, 2e partie: étude numérique", RAIRO Math. Model. Numer. Anal. 25, pp 535-560, 1991.

[2] Bardi M. and Falcone M., "Discrete approximation of the minimal time function for systems with regular optimal trajectories", Lecture Notes in Control and Informations Sciences, "Analysis and Optimization of systems", $n^{\circ} 114$, Springer-Verlag, 1990.

[3] Bardi M. and Falcone M., "An approximation scheme for the minimum time function", SIAM Journal, Control and Optimization, Vol 28, $n^{\circ} 4$, pp950-965, July 1990.

[4] Bardi M., Falcone M. and Soravia P., "Fully discrete schemes for the value function of pursuit-evasion games", 5 th International symposium in differential games and applications, Grimentz, Swizerland jully 1992.

[5] Bardi M. and Soravia P., "Approximation of differential games of pursuit-evasion by discrete time games", Differential Games - Developments in modelling and computation, R.P. Hämäläinen, H.K. Ehtamo Eds, Lecture Notes in Control and Information Sciences, Vol 156, Springer-Verlag, 1991.

[6] Barles G., Perthame B., "Discontinuous solutions of deterministic optimal stopping time problems" Mathematical Modelling and Numerical Analysis, Vol $21 \mathrm{n}^{\circ} 4$ pp 557-579, 1987.

[7] Bensoussan A., Lions J. L., "Contrôle impulsionnel et inequations quasi-variationnelles", Dunot, Paris, 1982.

[8] Camilli F., Falcone M., "An approximation scheme for the optimal control of diffusion processes" Report Università degli Studi di Roma "La Sapienza" 1992.

[9] Capuzzo Dolcetta I. "On a discrete approximation of the Hamilton-Jacobi equation of dynamic programming", Appl Math Optim 10, pp 367-377, 1983.

[10] Capuzzo Dolcetta I., Evans L. C., "Optimal switching for ordinary differential equations", SIAM Journal of Control and Optimization, Vol 22, pp 143-161, 1984.

$\mathrm{RR} \mathrm{n}^{\circ} 2926$ 
[11] Capuzzo Dolcetta I. and Ishii H., "Approximation solutions of the Bellman equation of deterministic control theory", Appl Math Optim 11, pp 161-181, 1984.

[12] Crandall M.G. and Lions P. L., "Viscosity solutions of Hamilton-Jacobi equations.", Trans AMS 277, pp 1-42, 1983.

[13] Ciarlet P. G., "Discrete maximun principle for finite-difference operators", Aequations Math. Vol 4, pp 338-352, 1970.

[14] Ciarlet P. G., Raviart P. A., "Maximun principle and uniform convergence for a finite elements method", Computer methods in Applied Mechanics and Engineering, Vol 2 pp. 17-31, 1973.

[15] Cortey-Dumond P., "Approximation numérique d'une inéquation quasi variationnelle liée à des problèmes de gestion de stock", R.A.I.R.O. Analyse numérique, Vol 14, $n^{0} 4$, pp. 335-346, 1980.

[16] Crandall M.G., Evans L. C. and Lions P. L., "Some properties of solutions of Hamilton-Jacobi equations", Trans AMS 282, pp 487-502, 1984.

[17] Crandall M.G., Lions P. L., "Two approximations of solutions of Hamilton-Jacobi equations", Math. Comp. 43, pp. 1-19, 1984.

[18] González R., "Sur la résolution de l'équation de Hamilton-Jacobi du contrôle déterministe", Cahiers de Mathématiques de la Desicion, $n^{0} 8029,8029$ bis. Ceremade Université de Paris-Dauphine, 1980.

[19] González R. and Rofman E., "On deterministic control problem - An approximation procedure for the optimal cost ", INRIA Report $n^{\circ} 151$.

[20] González R., Sagatizábal C., "Optimal control of arborescent multilevel inventory production system “, Report INRIA $n^{0}$ 1166, 1990.

[21] González R. and Tidball M., "On a discrete time approximation of the Hamilton-Jacobi equation", Report INRIA $n^{0}$ 1375, 1990.

[22] González R. and Tidball M., "On the rate of convergence of fully discrete solutions of Hamilton-Jacobi equation", Report INRIA $n^{0}$ 1376, 1991.

[23] González R. and Tidball M., "Sur l'ordre de convergence des solutions discrétisées en temps et en espace de l'équation de Hamilton-Jacobi", Comptes Rendus Acad. Sci. Paris, Tomo 314, Serie I, pp 479-482, 1992.

[24] Hanouset B, Joly J. L., "Convergence uniforme des iterés definisant la solution d'une inéquation quasi variationnelle abstraite", Comptes rendus Acad. Sc. Paris, Serie A, tome 286, pp. 735-738, 1978.

[25] Lions P.L., "Generalized solutions of Hamilton-Jacobi equations", Pitman London, 1982.

INRIA 
[26] Souganidis P.E., "Approximations schemes for viscosity solutions of Hamilton-Jacobi equations", J. Differential Equations 75, pp. 1-43, 1985.

[27] Strang G., Fix G., "An Analysis of the finite elements method", Prentice-Hall, Englewood Cliffs, NJ, 1973.

[28] Tidball M. and González R.L.V., "Zero sum differential games with stopping times. Some results about its numerical resolution", Proceedings of Fifth International Symposium on Dynamics Games and Applications, Grimentz, Swizerland, 15 - 18 july 1992. Annals of Dynamics games, Vol 1, 1993.

[29] Tidball M., "Sobre la resolución numérica de las ecuaciones de Hamilton- Jacobi-Bellman", Thesis, University of Rosario, 1991.

$\mathrm{RR} n{ }^{\circ} 2926$ 
Unité de recherche INRIA Lorraine, Technopôle de Nancy-Brabois, Campus scientifique, 615 rue du Jardin Botanique, BP 101, 54600 VILLERS LÈS NANCY

Unité de recherche INRIA Rennes, Irisa, Campus universitaire de Beaulieu, 35042 RENNES Cedex Unité de recherche INRIA Rhône-Alpes, 655, avenue de l'Europe, 38330 MONTBONNOT ST MARTIN Unité de recherche INRIA Rocquencourt, Domaine de Voluceau, Rocquencourt, BP 105, 78153 LE CHESNAY Cedex Unité de recherche INRIA Sophia-Antipolis, 2004 route des Lucioles, BP 93, 06902 SOPHIA-ANTIPOLIS Cedex

\section{Éditeur}

INRIA, Domaine de Voluceau, Rocquencourt, BP 105, 78153 LE CHESNAY Cedex (France)

ISSN 0249-6399 